\title{
Assignment of Dynamic Regions in Biological Solids Enabled by Spin-State Selective NMR Experiments
}

\author{
Rasmus Linser, Uwe Fink, and Bernd Reif* \\ Leibniz-Institut für Molekulare Pharmakologie (FMP), Robert-Rössle-St. 10, 13125 Berlin-Buch, Germany
}

Received March 29, 2010; E-mail: reif@fmp-berlin.de

Biological function largely depends on protein dynamics. Protein-protein interactions and ligand recognition have been shown to occur on a $\mu$ s time scale. ${ }^{1}$ In membrane proteins, the presence of slow motion has been a hurdle for X-ray crystallography, as in the case of the $\beta_{2}$-adrenergic G-protein coupled receptor (GPCR). ${ }^{2}$ Also for NMR spectroscopy, intermediate $(\mathrm{ns}-\mu \mathrm{s})$ motion tends to be an obstacle. In many amyloidogenic peptides and proteins, large parts of the primary sequence are often obscured and do not yield detectable resonances in solid-state NMR spectra, presumably due to dynamics. ${ }^{3}$ In the voltage gated membrane protein $\mathrm{VDAC},{ }^{4,5}$ large segments of the constricting $\mathrm{N}$-terminal $\alpha$-helix, which seems important for the gating process, could not be assigned due to dynamics. ${ }^{5}$ Ironically, those parts of a protein undergoing slow motion are particularly interesting for the understanding of its biology. We and others have demonstrated that sparsely protonated proteins can be successfully employed in Magic Angle Spinning (MAS) solid-state NMR for proton detection, ${ }^{6}$ INEPT based resonance assignment strategies, ${ }^{7}$ and mapping of solvent accessibility. ${ }^{8}$ This approach allowed us to show for the first time that mutual cancellation of dipole-dipole and chemical shift anisotropy (CSA) relaxation pathways ${ }^{9,10}$ can give rise to differential transverse relaxation times $T_{2}$ also in the solid state. While in the solution state, the effect is due to isotropic tumbling of the molecule, differential relaxation in the solid-state was shown to be due to internal mobility. ${ }^{11,12}$

We show here that TROSY ${ }^{10}$ type scalar coupling based triple resonance experiments in combination with perdeuteration are beneficial for the detection and assignment of those residues in the protein which undergo intermediate $(\mathrm{ns}-\mu \mathrm{s})$ dynamics. Standard (non-spin-state selective) triple resonance experiments, in contrast, perform extremely poorly in these cases. ${ }^{7}$ Conventional MAS solidstate NMR experiments that are based on dipolar transfers are shown not to detect these flexible residues at all.

Figures $1 \mathrm{~A}$ and $\mathrm{B}$ depict the first ${ }^{1} \mathrm{H} /{ }^{15} \mathrm{~N}$ planes of tripleresonance out and back $\mathrm{HNCO}$ experiments, in which ${ }^{1} \mathrm{H}$ magnetization is transferred to $\mathrm{C}^{\prime}$ and back. For these experiments, a sample of the chicken $\alpha$-spectrin SH3 is employed which is $100 \%$ perdeuterated at nonexchangeable sites and partially back-exchanged with protons at labile sites. ${ }^{7}$ Paramagnetic Relaxation Enhancement (PRE) using $\mathrm{Cu}$-edta was employed for accelerated data acquisition. ${ }^{13}$ While the spectrum in Figure $1 \mathrm{~A}$ was recorded using a standard HNCO pulse scheme, ${ }^{14}$ the spectrum in Figure 1B was recorded with spin-state selection, ${ }^{15}$ using the pulse schemes shown in Supplementary Figure $1 \mathrm{E}$ and $\mathrm{F}$, respectively. Even for the temperature which yields the best signal-to-noise ratio, residues located in mobile parts of the protein (labeled in black) have severely reduced intensities in comparison to residues that are situated in immobile $\beta$-sheet regions (labeled in gray). For those dynamic residues, selection of the slowly relaxing component turns out to be beneficial for obtaining intense ${ }^{1} \mathrm{H} /{ }^{15} \mathrm{~N} /{ }^{13} \mathrm{C}$-correlations.
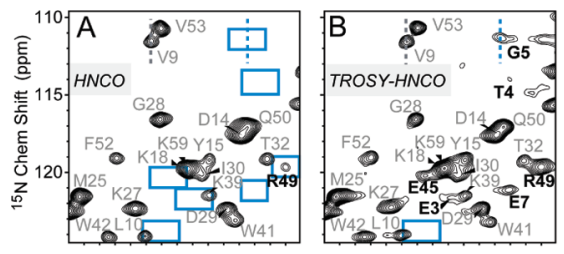

C
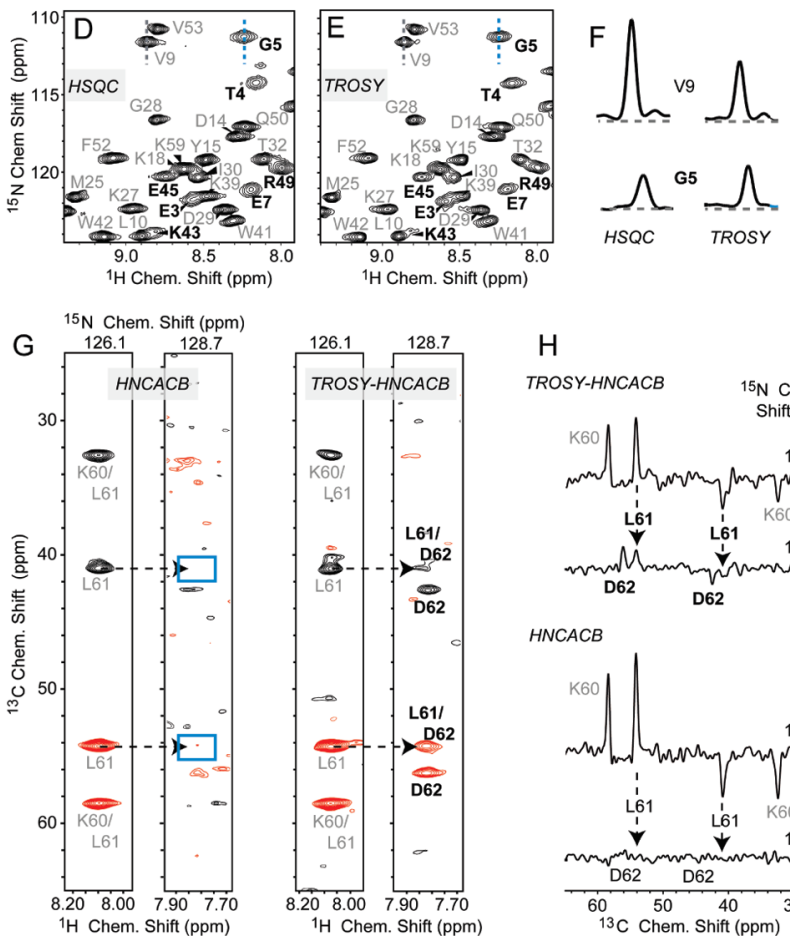

$\mathrm{H}$

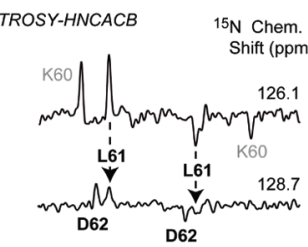

HNCACB

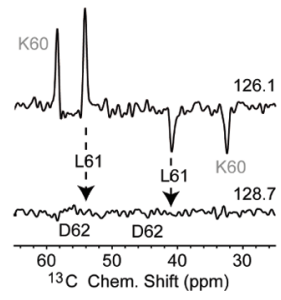

Figure 1. First $\mathrm{H} / \mathrm{N}$ plane of a standard 3D-HNCO (A) and a 3D-TROSYHNCO (B) experiment in the solid-state. Mobile residues are several fold enhanced using TROSY. A smaller gain is observed in double-resonance experiments $(\mathrm{D}, \mathrm{E})$ which are missing the N-CO INEPT periods. Cross peaks of mobile (rigid) residues are labeled in black (gray) letters. Blue boxes highlight the frequency of resonances which are below the lowest contour level. The experiments in $\mathrm{A} / \mathrm{B}$ and $\mathrm{D} / \mathrm{E}$, respectively, were recorded and processed under identical conditions and plotted to the same scale. The lowest contour level was chosen as $3 \sigma$ compared to noise rmsd. (Acquisition and processing parameters for the triple-resonance experiments in A and B were different to the parameters employed for the double resonance experiments in D and E). ( $\mathrm{C}$ and F) Cross sections at the ${ }^{1} \mathrm{H}$ resonance frequencies of V9 (rigid) and G5 (mobile). (G) Selected strips for a standard HNCACB and a TROSY-HNCACB taken at the amide nitrogen shift of L61 (rigid) and D62 (mobile), and respective cross sections $(\mathrm{H})$. Red and black colors refer to positive and negative contours. All spectra were recorded at a temperature of $22{ }^{\circ} \mathrm{C}$.

The absence of respective signals in the standard experiment is indicated with a blue rectangle (intensity is below the lowest contour level). For comparison, Figure 1D and E show selected regions of an HSQC and a TROSY H/N-correlation illustrating the intensity 
differences for flexible and immobile parts of the protein in doubleresonance experiments. Figure $1 \mathrm{C}$ and $\mathrm{F}$ show cross sections along the ${ }^{15} \mathrm{~N}$ dimension through the spectra in $\mathrm{A}, \mathrm{B}, \mathrm{D}$, and $\mathrm{E}$ for residues V9 and G5. For triple resonance experiments which are based on scalar ${ }^{15} \mathrm{~N} /{ }^{13} \mathrm{C}$ transfers, the benefits of spin state selection for flexible residues are clearly more pronounced in comparison to the $\mathrm{H} / \mathrm{N}$ double resonance correlations. Figure $1 \mathrm{G}$ and $\mathrm{H}$ show strips and cross sections extracted from a standard HNCACB and TROSY-HNCACB at the ${ }^{15} \mathrm{~N}$ chemical shift of L61 and D62. The sensitivity of weak peaks is clearly improved in the TROSY triple resonance experiments.

TROSY discards half of the starting magnetization but is "sensitivity enhanced", ${ }^{16}$ such that sensitivity is reduced by a factor of $\sqrt{ } 2$ in comparison to a standard experiment if no relaxation occurs. This disadvantage can in principle be circumvented using IPAP sequences. ${ }^{17}$ Nevertheless, for rigid residues with $R_{2}\left(\mathrm{~N}^{-} \mathrm{H}^{\alpha}\right)$ being comparable to $R_{2}\left(\mathrm{~N}^{-} \mathrm{H}^{\beta}\right)$, spin-state selection is disadvantageous. For residues undergoing intermediate time scale motion, however, the intensities observed in the TROSY experiments surmount the intensities achieved in a standard experiment. As expected, this effect is strongly temperature dependent (see Supplementary Figure 2). The signal-to-noise ratio as a function of amino acid sequence for the standard HNCO and the TROSYHNCO is shown in Supplementary Figure 3. In comparison to the standard HNCO experiment, signal-to-noise increases by a factor of $1.5,1.8,1.9$, and $\sim 3$ for $\mathrm{G} 5, \mathrm{E} 45, \mathrm{R} 49$, and E7, respectively, which corresponds to a factor of $2.3,3.2,3.6$, and 9 in measurement time. Comparable enhancement factors are found for other residues labeled in bold in Figure 1.
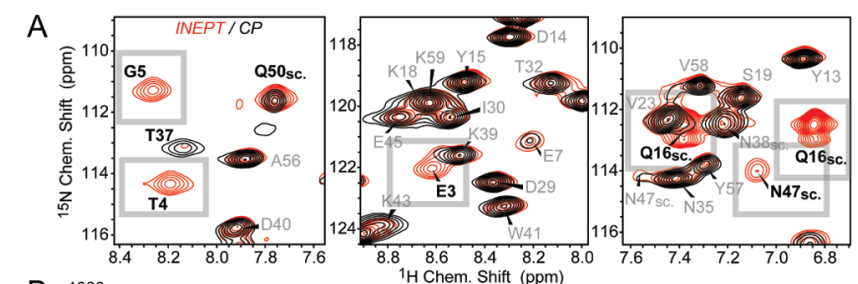

B

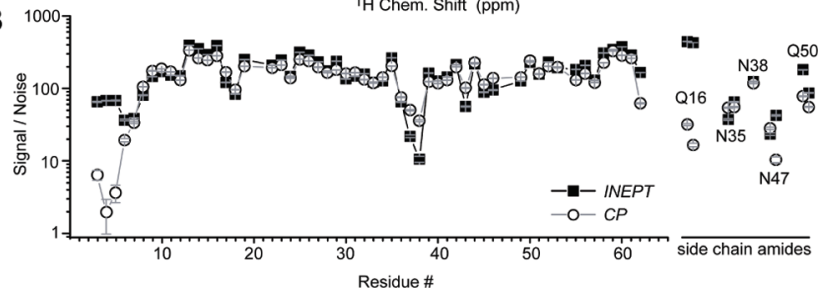

Figure 2. (A) $\mathrm{H} / \mathrm{N}$-correlation using INEPT (red) and CP (black) for magnetization transfer. INEPT transfer steps allow to observe resonances of mobile residues that are not detected using dipolar based magnetization transfers (highlighted by rectangular boxes). Both spectra were recorded, processed and plotted using identical parameters. The full spectrum is shown in Supplementary Figure 4. (B) Signal to noise ratio of CP (O) and INEPT (ם) based heteronuclear correlation experiments. Mobile residues, especially in the $\mathrm{N}$-terminus and side chain amides, are strongly attenuated in case $\mathrm{CP}$ is employed for magnetization transfer. In regular secondary structure elements, INEPT and CP yield comparable sensitivities. The experiements were recorded at a temperature of $22{ }^{\circ} \mathrm{C}$.

We have shown previously that there is slow motion in the solid state $^{18}$ and that these dynamics influence the line widths in MAS solid-state NMR experiments. ${ }^{19}$ At that time, only the C-terminal residue D62 was observed to undergo large amplitude fluctuation on a slow time scale in comparison to amide moieties within the rigid $\beta$-sheet structures. The previous experiments employed crosspolarization $(\mathrm{CP})$, which suppresses magnetization transfer for mobile residues. Thus, amide moieties which are particularly prone to differential relaxation were excluded from the analysis. Using scalar coupling based magnetization transfers, we find differential relaxation rates which are up to a factor of 2-3 times larger than what was observed in the previous study. ${ }^{11}$ This way, 10 additional residues are accessible which all have significant cross-correlated relaxation rates.

Figure 2 depicts a comparison of 2D H/N-correlations that were acquired using CP and INEPT for magnetization transfer, respectively. Resonances which are observable exclusively in the INEPT experiment originate from residues located in the $\mathrm{N}$-terminus as well as from side chain amides. These resonances appear to be broadened in the HSQC spectra, which is presumably due to large amplitude slow dynamics (see Supplementary Figure 5). Resonances in the n-Src loop (residues 36-39) show an increased signal-tonoise ratio in $\mathrm{CP}$ experiments, which is due to chemical exchange dynamics. The best signal-to-noise ratio for the dynamic residues is obtained at moderate temperatures $\left(22^{\circ} \mathrm{C}\right)$. A comparison of $\mathrm{H} / \mathrm{N}$ correlation spectra recorded at different temperatures is shown in Supplementary Figure 6.

To quantify differential relaxation, we used spin-state selective experiments. Figure $3 \mathrm{~A}$ shows the spectra obtained for the slowly and fast relaxing coherences $\mathrm{N}^{-} \mathrm{H}^{\alpha}$ and $\mathrm{N}^{-} \mathrm{H}^{\beta}$ of a representative flexible residue (G5) and an immobile residue (V53). In Figure $3 \mathrm{~B}$, the rates ${ }^{15} \mathrm{~N}-R_{2}\left(\mathrm{~N}^{-} \mathrm{H}^{\alpha}\right)$ and ${ }^{15} \mathrm{~N}-R_{2}\left(\mathrm{~N}^{-} \mathrm{H}^{\beta}\right)$ measured at 3 and $22{ }^{\circ} \mathrm{C}$ are represented as a function of the amino acid sequence (the employed pulse scheme is shown in Supplementary Figure 1C). In addition to the C-terminal D62, large differences between ${ }^{15} \mathrm{~N}$ $R_{2}\left(\mathrm{~N}^{-} \mathrm{H}^{\alpha}\right)$ and ${ }^{15} \mathrm{~N}-R_{2}\left(\mathrm{~N}^{-} \mathrm{H}^{\beta}\right)$ are found in the loop region around V46 (distal loop) and the N-terminal residues, which were not detected before. At $22{ }^{\circ} \mathrm{C}, \mathrm{N}^{-} \mathrm{H}^{\alpha}$ and $\mathrm{N}^{-} \mathrm{H}^{\beta}$ rates differ by up to a factor of $2-3$. At $3{ }^{\circ} \mathrm{C}$, differential relaxation for these residues is even larger. This observation is in agreement with an increased correlation time $\tau$ at low temperatures. ${ }^{20}$ For most residues located in the $\mathrm{N}$-terminus, resonance intensities are reduced to a value where a reasonable determination of $R_{2}$ is not possible anymore. For D62, differential relaxation at $3{ }^{\circ} \mathrm{C}$ compared to $22{ }^{\circ} \mathrm{C}$ is increased more than 3-fold. We observe the opposite behavior for the N-Src loop, which shows increased relaxation rates at higher temperatures. Resonances of this loop are hardly detectable at $22{ }^{\circ} \mathrm{C}$, which prevented the quantitative determination of relaxation rates in this case. We speculate that these residues undergo chemical exchange at higher temperatures. Interestingly, we find significant differential relaxation rates for residues $30-35$, even though the $\beta$-sheet motif would suggest low mobility. We have previously reported an alternating pattern of ${ }^{15} \mathrm{~N}-T_{1}$ relaxation times for this region. ${ }^{21}$ In comparison to previous results, ${ }^{11}$ we observe higher (absolute) $R_{2}$ rates. This is presumably due to the higher degree of protonation $(25 \%){ }^{22} R_{2}$ rates were not determined at temperatures higher than $22{ }^{\circ} \mathrm{C}$ in order not to compromise sample integrity.

The assignment of the N-terminus of the protein is summarized in Supplementary Figure 7. The assignments are supported with data obtained from ${ }^{1} \mathrm{H},{ }^{1} \mathrm{H}$ spin diffusion experiments and by comparison with solution-state chemical shifts, ${ }^{23}$ which match the solid-state chemical shifts reasonably well. ${ }^{24}$ Despite the use of TROSY experiments, assignment of mobile residues is still not trivial due to low signal-to-noise, and dynamic residues display a ca. 2-8-fold lower intensity compared to rigid residues. We expect, however, that larger gains in sensitivity are possible through spinstate selection when higher magnetic fields become available for solid-state NMR.

In summary, we report a strategy to detect and assign regions in a solid protein that undergo intermediate time scale dynamics. Using spin-state selection in MAS solid-state NMR in combination with 


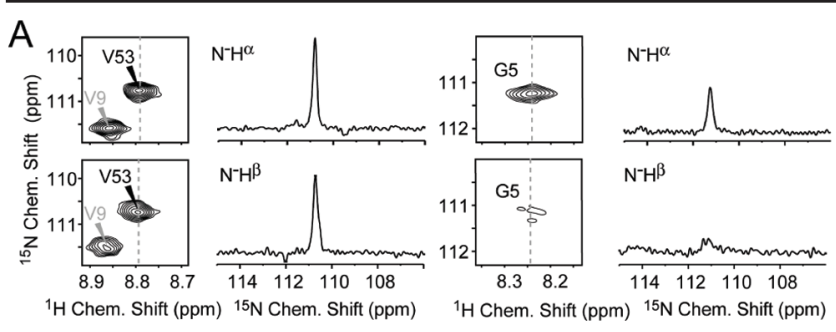

B
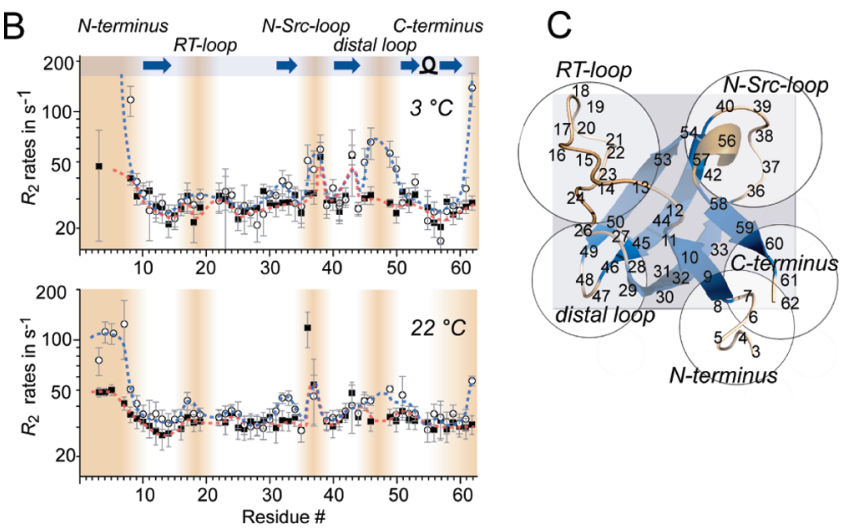

Figure 3. (A) Slowly $\left(\mathrm{N}^{-} \mathrm{H}^{\alpha}\right)$ and fast $\left(\mathrm{N}^{-} \mathrm{H}^{\beta}\right)$ relaxing spin states in the solid state. A large differential intensity is indicative for large amplitude slow motion on a ns $-\mu$ s time scale. While immobile residues (represented by V53, left) do not display pronounced differential relaxation, residues in flexible regions (G5, right) show a substantial difference in intensity for the coherences $\mathrm{N}^{-} \mathrm{H}^{\alpha}$ and $\mathrm{N}^{-} \mathrm{H}^{\beta}$. Separation of spin states is achieved by employing the pulse scheme depicted in Supplementary Figure 1C. 1D traces represent slices along the ${ }^{15} \mathrm{~N}$ dimension (dashed lines). The spectra were recorded at $22{ }^{\circ} \mathrm{C}$ and plotted identically and without use of apodization. (B) Relaxation rates for coherences $\mathrm{N}^{-} \mathrm{H}^{\alpha}$ (black squares) and $\mathrm{N}^{-} \mathrm{H}^{\beta}$ (white circles). Data were recorded for 3 and $22^{\circ} \mathrm{C}$. Dashed lines are drawn to guide the eye. The secondary structure is indicated in the upper part of the figure. Loop regions are shaded in brown. Protein internal motion and the effect of differential relaxation are site specific and temperature dependent. Especially high values for differential relaxation are found for D62, the $\mathrm{N}$-terminus, and the distal loop involving residues 45 to 50 . At low temperature, relaxation rates were not measurable for the $\mathrm{N}$-terminal residues due to insufficient signal-to-noise. In contrast to CP based magnetization transfer, ${ }^{11} 10$ more amide moieties with significant cross-correlated relaxation rates can now be characterized. (C) Structural representation of the $\alpha$-spectrin SH3 domain. ${ }^{19}$ The flexible N-terminus (up to K6) is not refined in the crystal structure and is shown with an arbitrary conformation.

$J$-based magnetization transfer steps, we observe mobile regions at the termini and in a loop region of the microcrystalline $\alpha$-spectrin SH3 domain which have been invisible so far using conventional techniques. TROSY type techniques significantly improve the spectral quality of ${ }^{1} \mathrm{H} /{ }^{15} \mathrm{~N} /{ }^{13} \mathrm{C}$-correlations for residues undergoing intermediate time scale local motions. Although only a few residues in the SH3 domain could be newly assigned, the study might be an exemplary case for the investigation of flexible loops of membrane proteins or amyloid fibrils. For the understanding of protein function, in particular dynamic regions will have to be characterized and cannot be excluded from the assignment process.
Extensive deuteration is mandatory for this approach as otherwise spin states cannot be separated easily. We assume that these techniques become even more important if higher MAS frequencies and larger magnetic fields become available in the future.

Acknowledgment. This work is dedicated to Professor Horst Kessler on the occassion of his 70th birthday, and to Professor Christian Griesinger on the occassion of his 50th birthday. This research was supported by the Leibniz-Gemeinschaft and the DFG (Re1435, SFB449, SFB740). R.L. is a Kekulé scholar and acknowledges financial support by the Verband der Chemischen Industrie (VCI).

Supporting Information Available: Experimental details, quantitative data comparison, assignments. This material is available free of charge via the Internet at http://pubs.acs.org.

\section{References}

(1) Lange, O. F.; Lakomek, N.-A.; Fares, C.; Schroeder, G. F.; Walter, K. F. A.; Becker, S.; Meiler, J.; Grubmueller, H.; Griesinger, C.; Groot, B. L. d. Science 2008, 320, 1471-1475.

(2) Rosenbaum, D. M.; Cherezov, V.; Hanson, M. A.; Rasmussen, S. G. F.; Thian, F. S.; Kobilka, T. S.; Choi, H.-J.; Yao, X.-J.; Weis, W. I.; Stevens, R. C.; Kobilka, B. K. Science 2007, 318, 1266-1273.

(3) Andronesi, O. C.; Becker, S.; Seidel, K.; Heise, H.; Young, H. S.; Baldus, M. J. Am. Chem. Soc. 2005, 127, 12965-12974. Siemer, A. B.; Arnold, A. A.; Ritter, C.; Westfeld, T.; Ernst, M.; Riek, R.; Meier, B. H. J. Am. Chem. Soc. 2006, 128, 13224-13228. Helmus, J. J.; Surewicz, K.; Nadaud, P. S.; Surewicz, W. K.; Jaroniec, C. P. Proc. Natl. Acad. Sci. U.S.A. 2008, 105, 6284-6289.

(4) Bayrhuber, M.; Meins, T.; Habeck, M.; Becker, S.; Giller, K.; Villinger, S.; Vonrhein, C.; Griesinger, C.; Zweckstetter, M.; Zeth, K. Proc. Natl. Acad. Sci. U.S.A. 2008, 105, 15370-15375.

(5) Hiller, S.; Garces, R. G.; Malia, T. J.; Orekhov, V. Y.; Colombini, M.; Wagner, G. Science 2008, 321, 1206.

(6) Chevelkov, V.; Rehbein, K.; Diel, A.; Reif, B. Angew. Chem., Int. Ed. 2006, 45, 3878-3881. Zhou, D. H.; Shea, J. J.; Nieuwkoop, A. J.; Franks, W. T.; Wylie, B. J.; Mullen, C.; Sandoz, D.; Rienstra, C. M. Angew. Chem., Int. Ed. 2007, 46, 8380-8383. (b) Agarwal, V.; Reif, B. J. Magn. Reson. 2008, 194, 16-24.

(7) Linser, R.; Fink, U.; Reif, B. J. Magn. Reson. 2008, 193, 89-93.

(8) Chevelkov, V.; Faelber, K.; Diehl, A.; Heinemann, U.; Oschkinat, H.; Reif, B. J. Biomol. NMR 2005, 31, 295-310. Linser, R.; Fink, U.; Reif, B. J. Am. Chem. Soc. 2009, 131, 13703-13708.

(9) Gueron, M.; Leroy, J. L.; Griffey, R. H. J. Am. Chem. Soc. 1983, 105, 7262-7266.

(10) Pervushin, K. V.; Riek, R.; Wider, G.; Wüthrich, K. Proc. Natl. Acad. Sci. U.S.A. 1997, 94, 12366-12371.

(11) Chevelkov, V.; Diehl, A.; Reif, B. Magn. Reson. Chem. 2007, 45, S156160.

(12) Skrynnikov, N. R. Magn. Reson. Chem. 2007, 45, S161-S173.

(13) Linser, R.; Chevelkov, V.; Diehl, A.; Reif, B. J. Magn. Reson. 2007, 189, 209-216.

(14) Grzesiek, S.; Bax, A. J. Magn. Reson. 1992, 96, 432-440.

(15) Yang, D.; Kay, L. E. J. Biomol. NMR 1999, 13, 3-10.

(16) Palmer, A. G.; Cavanagh, J.; Wright, P. E.; Rance, M. J. Magn. Reson. 1991, 93, 151-170.

(17) Ottiger, M.; Delaglio, F.; Bax, A. J. Magn. Reson. 1998, 131, 373-378.

(18) Chevelkov, V.; Fink, U.; Reif, B. J. Biomol. NMR 2009, 45, 197-126.

(19) Chevelkov, V.; Faelber, K.; Schrey, A.; Rehbein, K.; Diehl, A.; Reif, B. J. Am. Chem. Soc. 2007, 129, 10195-10200.

(20) Clore, G. M.; Szabo, A.; Bax, A.; Kay, L. E.; Driscoll, P. C.; Gronenborn, A. M. J. Am. Chem. Soc. 1990, 112, 4989-4991.

(21) Chevelkov, V.; Diehl, A.; Reif, B. J. Chem. Phys. 2008, 128, 052316.

(22) Akbey, Ü.; Lange, S.; Franks, W. T.; Linser, R.; Rehbein, K.; Diehl, A.; van Rossum, B.-J.; Reif, B.; Oschkinat, H. J. Biomol. NMR 2009, 46, 6773.

(23) Blanco, F. J.; Ortiz, A. R.; Serrano, L. J. Biomol. NMR 1997, 9, 347-357.

(24) van Rossum, B.-J.; Castellani, F.; Rehbein, K.; Pauli, J.; Oschkinat, H. ChemBioChem 2001, 2, 906-914.

JA102612M 